\title{
The Possibilities of Transcribing Orchestral Works Subject to Essentiality of their Original Orchestration
}

\author{
Rytis Urniežius / rytisur@gmail.com \\ Šiauliai University, LT
}

\begin{abstract}
The relationship of orchestration with other means of musical expression and its influence upon the possibilities of transcribing musical works are analysed in this article. The criteria of transcribing symphonic music for the wind band are considered. The demand for the quality of transcriptions induces to ground the criteria of transcribing. The role of the original orchestration in a particular piece of music is an especially important criterion. One of the main conditions determining the quality of transcriptions is the adequacy of original orchestration and the orchestration of the transcription. It was deduced that the relationship between orchestration and the rest of the musical material might be immanent, essential or auxiliary, inessential. According to the type of this relationship, a different approach to the transcribing should be applied in every particular case. The comprehensive cognition of original orchestration and pursuing the adequacy of timbres in transcriptions limit the quantity of the compositions suitable for transcribing and determine the possibilities of its interpretation.
\end{abstract}

\section{Keywords}

orchestration, transcription, timbre, symphony orchestra, wind band, adequacy 
Orchestration is the mean of musical language to which musicologists give comparatively little attention. The reasons of this phenomenon are different. One of them is the inclination of the researchers to derogate the orchestral factor in the totality of the means of musical expression. Therefore, it is reasonable to agree with these authors who indicate that the orchestration has been undeservedly rarely analysed for many years, its importance for the artistic expression of the musical composition was underestimated and most researchers confine their attention to "predominantly monochrome note-heads of the short score". ${ }^{1}$ On the other hand, it was many times noticed that the neglecting of the orchestration may impoverish the analysis of a musical piece, whereas the observant approach to the orchestration can even change the perception of the creation's structure. However, some serious research works including doctoral dissertations on the subject of orchestration written in recent years can be found while searching in the electronic databases; therefore, it seems that this field of investigation increasingly attracts attention of the researchers.

Importance of the orchestration among other components of musical language becomes an especially topical in case changing its existing version - transcribing or arranging it for another set of instruments. Timbre in this case must be inevitably changed (besides different solutions of modifying the texture). Namely in this situation the connections of the orchestration with the whole remaining structural material of a musical creation becomes especially obvious. The subject of this article is the peculiarities of the instrumental embodiment of musical creative pieces and their influence upon the possibilities of transcribing these pieces for another kind of a performing medium. The aim of the article is to examine the significance of orchestration in the whole spectrum of the means of musical expression from the viewpoint of their transcribing for another medium.

The article deals with the criteria of transcribing symphonic music for the wind band. This type of transcribing has been chosen firstly because it can be considered as one of the most common fields of transcribing musical pieces. The concert art music repertoire of the wind band consisted almost exclusively of the transcriptions up to the middle of the twentieth century. During the twentieth century and especially its second half the original compositions for the wind band became predominant in the repertoire of the bands in most Western countries; however, the transcriptions still occupy a significant part of their repertoire. Transcribed art music creations are included in the repertoire of the educational institutions as well as professional bands. It is reasonably considered that the performing of the works by classics is very important for educating band members and it also enhances the level of band's excellence. Therefore, the quality of transcriptions, their artistic value is an extremely important issue. During the whole history of transcribing symphonic music for the wind band a lot of scores of very different quality have been created. Some of them were totally unsatisfactory from the point of view of

1 STOCK, Jonathan P. J. Orchestration as Structural Determinant: Mozart's Deployment of Woodwind Timbre in the Slow Movement of the C Minor Piano Concerto K. 491. Music Ẽ Letters, 1997, vol. 78, no. 2 (May), p. 210. 
their artistic quality. ${ }^{2}$ The increased demand for the quality of transcriptions in recent time raises the problem of the criteria which could serve as landmarks for the transcribers. It could be presumed that the adequacy of the timbres between the original score and the transcription should be one of the most important conditions which determine the quality of the transcriptions. Another reason of choosing this particular type of transcriptions is that both performing media - the symphony orchestra and the wind band - are the richest media considering the variety of timbres.

This research is not intended to thoroughly and comprehensively disclose all sides of the timbre influence upon the transcribing. Its task is to establish the theoretical background. The insights presented in this article could prompt further investigations in this field. Also, we believe that there are possibilities to apply the results of this research in practice of the transcribers' creative work and to implement them in the courses taught in music education institutions.

The adaptation of musical works for another instrumentation practices most likely existed since ancient times. It is also likely that in all times there were frictions between the supporters of such adaptation and purists who claimed that the original version of the piece was sacrosanct and outsiders should not be allowed to change it. ${ }^{3}$ However, practices of transcribing are so commonplace in our time that the question if they are admissible should not be raised. From our point of view another question is topical: what are the conditions of the re-composing of a musical piece which allow creating the artistically valuable secondary product?

A different instrumental interpretation of the musical work is possible because of the specificity of timbre in comparison with other means of musical expression. The acoustical nature of the sound has been discovered long time ago and now researchers are able to examine the spectra of the sounds applying the newest technologies. However, in the practice of musical performing, learning and cognition timbre is usually defined as a sensually perceived colour of the sound. The logical systematic relationship among musical timbres can hardly be established; therefore, it is impossible to create consequent scales of timbres, as it is possible in the case of a sound pitch, rhythm and (very approximately) dynamics. That is why the timbre can be separated from the structure of a pitch and rhythm, the essential components of music itself, thus creating the conditions to interpret this structure differently by changing the character of its timbres. It might appear that the indifference of the "pure music" to the orchestration is universal: the changes in one of the fundamental elements of musical language (melody, harmony, rhythm) would cause the changes of the very essence of a musical creation and even make music unrecognisable; whereas the changes of the orchestration in most cases can only enrich or impoverish a musical image but cannot change the essence of its musical ideas. This viewpoint is somewhat reasoned. The existence of transcriptions (sometimes artistically highly valuable), the methods of teaching orchestration which are based on

2 See URNIEŽIUS, Rytis. Transcriptions of the Symphonic Music for the Wind band: The Facets of Two Traditions [in Lithuanian and English]. Kürybos erdvès / The Spaces of Creation, 2015, vol. 21, p. 8-23.

3 BUTTERWORTH, Arthur. Reverse Transcription - A Matter of Black and White? [online]. 2004. [cit. 2016-2903]. URL: 〈http://www.musicweb-international.com/classrev/2004/Oct04/reverse_transcription.htm>. 
the scoring music written for another medium and other similar facts confirm the ambiguous and unstable relationship of the musical material with its instrumental embodiment thus authorising the existence of musical works in different instrumental versions. This is how the possibility of transferring a musical piece from one timbre context to another is validated.

On the other hand, such viewpoint should not be regarded unreservedly. It reflects just one - humble and auxiliary - role of the orchestration in music. If we examine the history of European orchestral music more attentively, we could easily find that the orchestration is not always a secondary and subsidiary element in point of other components of the musical language. Sometimes it can be very tightly connected with the melody, harmony, rhythm and dynamics, and it consequently becomes inseparable from the very essence of musical ideas. Therefore, two attitudes towards the role of the orchestration in a musical creation are possible: 1) the orchestration is an inessential, auxiliary attribute of a musical creation; b) orchestration is an essential, immanent attribute of a musical creation. Both attitudes could be treated as reasoned each in its own way. They reflect two different extremities of the same phenomenon.

It can be presumed that orchestration is more often treated as an inessential and auxiliary means of a musical expression able only to "tinge" the musical idea by means of a sound of one or another instrument. The second attitude which emphasises the immanent role of orchestration was properly described by Nikolai Rimsky-Korsakov in his treatise Principles of Orchestration: "Many people express an erroneous understanding saying that composer masterly scores or that composition is masterly orchestrated. The orchestration is a part of the very soul of creation. The composition itself is initially conceived for the orchestra and from this inception it anticipates certain orchestral colours peculiar only to this very piece and its creator. Is it possible to separate the essence of Wagner's music from his orchestration? It would sound similarly as if saying: that picture was masterly coloured by that artist". ${ }^{4}$ Arthur Butterworth noticed: “[...] some people are curious to know whether a composer also thinks of instrumental timbre at the outset. Does the melody come to the imagination already clothed in a particular instrumental or vocal colour? [ ...] it seems not improbable that the timbre of a melody comes along with an initial idea itself." 5 These authors claim that in the case of immanent orchestration the composers do not create a musical text separately in order to transfer it later to the orchestra. On the contrary, a particular element of the musical text is conceived in a certain timbre from the very beginning, thus becoming an inseparable part of the composer's initial idea (of course, it could be more or less adjusted later). In this case it is impossible to imagine the sounding of music abstracted from its timbre environment - the timbre, sound colour compound the indispensable part of the musical phenomenon. There are a lot of examples of symphonic and operatic music existing in the consciousness of a listener together with the unique timbre assigned by the com-

4 РИМСКИЙ-КОРСАКОВ, Николай. Основы оркестровки. Государственное музыкальное издательство. Москва, Ленинград, 1946 [Rimsky-Korsakov Nikolai. Principles of Orchestration. State Musical Publishing Office. Moscow, Leningrad, 1946. In Russian], p. 8.

5 BUTTERWORTH, Arthur. Transcriptions - a Change of Colour? [online]. 2001. [cit. 2016-03-29]. URL: 〈http://www.musicweb-international.com/ButterworthWrites/transcriptions.htm>. 
poser. For instance, the image of a sighing forest in Der Freischütz or Siegfried could not be conveyed by brass instruments. Meanwhile, it is difficult to imagine a fanfare theme in some pompous scene of an opera assigned for string instruments or flutes. Of course, string instruments are able to perform such theme without any technical difficulties, but the themes of such a character are unrepresentative of them.

In the case of immanent orchestration the embodiment of music in timbres is a stage of the creative process which is performed not as a distribution of completed musical material for instruments and their groups but moulded indistinguishably from other means of expression. Moreover, this embodiment often influences the character of these means. It might happen that a similar musical text (e. g. repeating of exposition material in recapitulation), when transferred to another instrumental context, undergoes changes because of accommodation to the new timbre and technical peculiarities of different instruments. To be precise, such text does not accommodate to a certain instrument; however, they both are shaped in a close unity. Thus, orchestration and thematic material exist in a unified whole, and, what is more, it may influence the form of a musical work. "Instrumentation is not simply the vessel into which some irreducible musical essence is poured. In the hands of certain composers, a timbre can be activated as a primary determinant of the musical structure". ${ }^{6}$

In the preface to his Principles of Orchestration, Rimsky-Korsakov notes that there are cases when the meaning and mood of a musical passage are so clear and unquestionable that nobody can doubt about the way of their orchestration. The composer confirms that a tremolo chord undoubtedly will go to string instruments, whereas a fanfare will be given to brass but not vice versa. However, secondary and easier solvable questions arise: is it necessary to double the tremolo with extended chords of woodwinds? For which instrument does the tessitura of the fanfare fit best of all? Does a solo instrument or unison fit better? Consequently, the musical material intended for a certain instrument should correspond to its nature.

Two aspects of this phenomenon should be distinguished: timbre as a quality of sound and technical peculiarities of the instrument. These two aspects affect musical material not separately but most often in unity. In a particular work of music, a text could be naturally connected with technical peculiarities of instruments (one of the reliable indicators - the convenience for musicians to perform the assigned musical material on their instruments). Simultaneously the material amalgamates with the timbre of instruments. Therefore, it is impossible to separate these two aspects. When symphonic works are transcribed for another medium, the peculiarity of timbre as well as technical characteristics of instruments should be considered. They determine the necessity of adequacy in transcribing. Of course, it is impossible to encompass all possible conditions of immanence (or non-immanence). For instance, there are special cases when a composer deliberately writes a part for the inconvenient or poorly sounding register of the instrument, thus striving to achieve an unexpected acoustical result: parody, comical programmatic

6 STOCK, op. cit., p. 219.

7 РИМСКИЙ-КОРСАКОВ, ор.cit., p. 80. 
The Possibilities of Transcribing Orchestral Works Subject to Essentiality of their Original ...

musical image etc. Gustav Mahler stated: "I often make the basses and bassoon squeak on the highest notes, while my flute huffs and puffs down below". ${ }^{8}$

Rimsky-Korsakov's compositions are a demonstrative example of such organic connection between the musical text and orchestration. While examining his scores, there is no necessity to look at the names of instruments in order to identify the parts of a certain instruments and groups of instruments relying on signs which prompt particular timbres: melody line, specific intervals, register, texture and other features (Ex. 1). Arthur Butterworth says: "[...] even the most casual glimpse of the scores of - say - Richard Strauss or Shostakovich, will reveal the marked differences between what the composer demands from the voices compared with that required of the strings, the wind or the percussion." 9

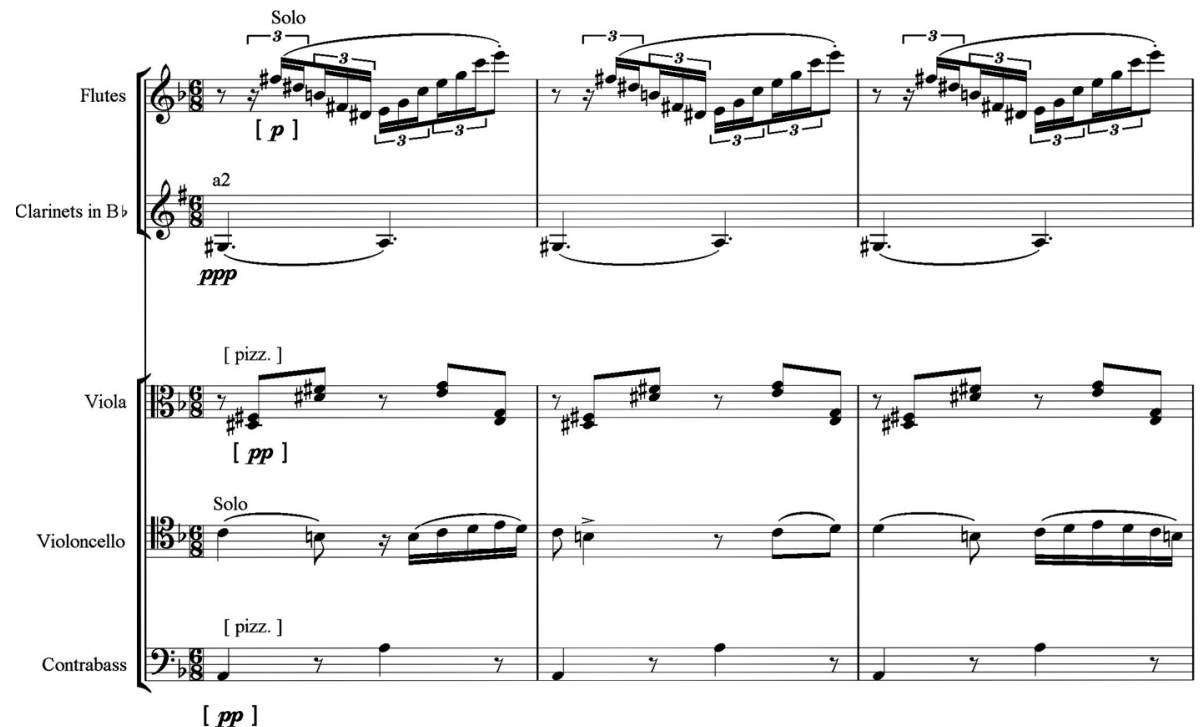

Ex. 1 Rimsky-Korsakov, Capriccio Espagnol, Movement IV. The melody of a solo cello is distinguished in the general context of all instruments thanks to the tension of its high register and warm, melodious timbre of A string. The accompaniment of pizzicato double basses and divided violas is light, containing octave leaps easily performed by string instruments. The clarinets pedal is in their low register; clarinets are able to perform piano even their lower notes effortlessly. The timbre of these sounds provides a darker colour to the passage. The agile figuration is very specific to the flute which demonstrates the sounding of all of its registers in this passage. The overall character of the chamber texture is lucid and the peculiarities of individual timbres are distinguished. The nature of every part corresponds to the specificity of technical abilities of the instruments. Each part has a characteristic rhythmic

weave. The instrumental lines are well-balanced and do not impede each other.

8 JOHNSON, Julian. Mahler's Voices. Expression and Irony in the Songs and Symphonies. Oxford University Press, 2009, p. 37.

9 BUTTERWORTH, Arthur. Transcriptions - a Change of Colour? [online]. 2001. [cit. 2016-03-29]. URL: <http://www.musicweb-international.com/ButterworthWrites/transcriptions.htm>. 
Thus, the role of orchestration might be very different. Inessential, auxiliary orchestration just characterises a musical text. Inherent, essential orchestration's role is determinant. It includes specificity of musical instruments into the structural nature of musical ideas during the stage of their inception. The dominance of one or another type of orchestration depends on composer's style and conception of a particular creation. In earlier times as well as in our days there are composers who do not use vivid colours in their compositions. Nevertheless, it would be overbold to say that such composers do not appreciate the role of orchestration or they are ignorant in its usage, just their way of musical thinking is different: "Many of them perhaps know orchestration even better that some colourists. Is it believable that Brahms is incompetent in orchestration? Vivid and pictorial sounding is absent in Brahms's works therefore the very method of his composing does not demand to strive for such sounding." ${ }^{10}$

Relying on the discussed connection of orchestration and musical material, it is possible to estimate the possibilities of transcribing the musical compositions for another medium. It is obvious that in the case of immanent original orchestration a transcriber should deeply analyse the relationship between an original musical text and an original orchestration in order to ensure that the result of transcribing will not distort the important features of musical ideas. There is no reason to transcribe the compositions in which orchestration is emphatically immanent and the nature of the means of the musical expression is inseparably connected with the specificity of the symphony orchestra: it is impossible to escape distortions of musical ideas while transferring their material to another set of instruments. The most important obstacle of transcribing symphonic music for the wind band is specificity of the string instruments parts. However, even the parts of wind instruments should not be copied mechanically: the functions of analogous orchestral groups in the symphony orchestra and the wind band are not the same. After exploiting the possibilities of the wind instruments of the wind band for the material performed by the wind instruments in the original score, the transcriber would have no more resources to compensate string instruments' parts.

Meanwhile the compositions whose orchestration is not immanent may be more or less successfully transcribed for another medium. The musical piece could be changed outwardly but its essence would remain intact. In this case the task of a transcriber is to convey the content of the musical text, whereas the orchestration just characterises it. Such pieces are either composed regardless of any instrumental specificity or were initially written for the piano and only later orchestrated for the symphony orchestra.

It is clear that the features of orchestration which are described here determine the choice of creations to be transcribed. The more the orchestration of the piece is inherently connected with its musical text the less the composition is suitable for transcribing. In cases when the timbre and technical specificity of instruments are not inherently connected with the text, the overall artistic conception usually remains intact (of course, if we assume that a transcriber will perform the work responsibly and creatively). Such are two extremes of orchestration possibilities. But in each particular case the role of

10 РИМСКИЙ-КОРСАКОВ, op. cit., p. 8-9. 
orchestration does not belong to either of extremes. There are a lot of compositions in which orchestration is not yet immanent but has already stepped over the boundary of the auxiliary type. Moreover, the features of immanent orchestration could be present only in a short passage of the piece; therefore, sometimes the transcribing is impossible only because of several measures which in no way could be conveyed by different means.

\section{Conclusions}

It can be presumed that transcribers should be obliged to strive for the quality of transcriptions which correspond to the artistic level of the original. Many famous musicians emphasised the necessity of the responsible attitude towards the quality of transcriptions. The composer and conductor W. Francis McBeth assumed that "transcribing orchestral music is a very touchy proposition, because it is my belief that if a transcription injures the original orchestration, it should not be done". ${ }^{11}$ This point of view impels to recognise that the quantity of symphonic compositions suitable for arranging is limited and one of the main criteria of their selection should be a character of original orchestration. On the grounds of analysis presented here, all orchestral compositions could be classified into three categories (strict boundaries could not be drawn between them) according to the possibilities of transcribing:

1. Creations of the auxiliary, inessential orchestration. Their musical text is composed regardless the peculiarities of the specificity of instruments. The choice of timbres while transcribing is fairly free. The transcriber possesses extensive possibilities for the creative work.

2. Creations of the transitional type. Their musical text is composed more or less regarding the peculiarities of the instruments' specificity. The transcriber's task is to convey adequately the original orchestral conception using available means.

3. Creations of the immanent, essential orchestration. Their musical text is inherently connected with original timbres and most often with the technical peculiarities of instruments. The transcribing is not recommended in most cases.

On the grounds of the scale of orchestration significance levels presented here, it is possible to draw approximate boundaries inside which the transcribing could be permissible. It could be presumed that the transcribing of the compositions which belong to the second and perhaps the third category would be acceptable if it answers the requirements of the adequacy of timbres. Some particular details of this adequacy could be defined: 1) original and alternative instruments should be of the same tessitura; 2) the registers of instruments should be the same or very similar; 3) other features which are generally dimensionless (richness, sharpness, poignancy etc.) should be transferred as adequately as possible. Among them the tension of timbre which determines prominence of different layers of texture should be especially emphasised. It is, of course,

11 See HOUSER, Russel J. An Examination of Wind Band Transcriptions. Thesis for the Degree of Master of Music. The University of Texas at El Paso, 2008, p. 5. 
impossible to retain the same level of adequacy at all levels of texture. Usually, the main attention is paid to the most prominent, characteristic elements (e. g. melody or countermelody).

More detailed and comprehensive identification of the adequacy conditions could become a subject of further research and formulating of practical recommendations. The adherence to these recommendations in the practice of transcribing could help to ensure the quality of transcriptions and at least partly preclude the negative criticism of the particular transcriptions and the practice of transcribing in general.

\section{Bibliography}

BUTTERWORTH, Arthur. Reverse Transcription - A Matter of Black and White? [online]. 2004. [cit. 2016-29-03]. URL: <http://www.musicweb-international.com/classrev/2004/Oct04/reverse_ transcription.htm $>$.

BUTTERWORTH, Arthur. Transcriptions - a Change of Colour? [online]. 2001. [cit. 2016-03-29]. URL: 〈http://www.musicweb-international.com/ButterworthWrites/transcriptions.htm>.

JOHNSON, Julian. Mahler's Voices. Expression and Irony in the Songs and Symphonies. Oxford University Press, 2009.

HOUSER, Russel J. An Examination of Wind Band Transcriptions. Thesis for the Degree of Master of Music. The University of Texas at El Paso, 2008.

STOCK, Jonathan P. J. Orchestration as Structural Determinant: Mozart's Deployment of Woodwind Timbre in the Slow Movement of the C Minor Piano Concerto K. 491. Music E Letters, 1997, vol. 78, no. 2 (May,), p. 210-219.

URNIEŽIUS, Rytis. Transcriptions of the Symphonic Music for the Wind band: The Facets of Two Traditions [in Lithuanian and English]. Kürybos erdves / The Spaces of Creation, 2015, vol. 21, p. 8-23.

РИМСКИЙ-КОРСАКОВ, Николай. Основы оркестровки. Государственное музыкальное издательство. Москва, Ленинград, 1946 [Rimsky-Korsakov Nikolai. Principles of Orchestration. State Musical Publishing Office. Moscow, Leningrad, 1946. In Russian] 
